Rapport - Société canadienne d'histoire de l'Église catholique

\title{
Le Comité des Fondateurs de l'Église canadienne
}

\section{Jacques Séguin}

Volume 31, 1964

URI : https://id.erudit.org/iderudit/1007342ar

DOI : https://doi.org/10.7202/1007342ar

Aller au sommaire du numéro

Éditeur(s)

La Société canadienne d'histoire de l'Église catholique

ISSN

0318-6148 (imprimé)

1927-7075 (numérique)

Découvrir la revue

Citer cet article

Séguin, J. (1964). Le Comité des Fondateurs de l'Église canadienne. Rapport -

Société canadienne d'histoire de l'Église catholique, 31, 23-29.

https://doi.org/10.7202/1007342ar

Tous droits réservés @ La Société canadienne d'histoire de l'Église catholique, 1965
Ce document est protégé par la loi sur le droit d'auteur. L’utilisation des services d'Érudit (y compris la reproduction) est assujettie à sa politique d'utilisation que vous pouvez consulter en ligne.

https://apropos.erudit.org/fr/usagers/politique-dutilisation/ 


\title{
Le Comité des Fondateurs de l'Église canadienne
}

\begin{abstract}
L'auteur analyse le mandement publié par les Evêques le 25 juillet 1942 sur les Pionniers de l'Eglise du Canada. Il nous introduit au Comité des Fondateurs de l'Eglise canadienne composé de dix-sept membres sous la présidence de $M$. le Chanoine Lionel Groulx. Le but de ce Comité est de faire connaître les vertus et les travaux des fondateurs, notamment ceux de $M^{g r}$ de Laval, de Marie de l'Incarnation, de Catherine de Saint-Augustin et de Marguerite Bourgeoys, de Jeanne Mance et de Marguerite d'Youville, par la prière, la propagande sous toutes ses formes en vue de promouvoir la canonisation de ces saints personnages de l'épopée mystique de nos origines.
\end{abstract}

Vous avez sans doute fait connaissance avec nos Pionniers de la foi bien avant que j'aie lu leur biographie. Je n'aurai donc pas à remplir aujourd'hui la tâche d'un sacristain qui trouve des chefs-d'œuvre oubliés dans une armoire, les époussette et se voit en présence d'inestimables tableaux d'art religieux. J'emprunte cette comparaison à $\mathrm{M}$. le chanoine Lionel Groulx, président du Comité des Fondateurs. Il en est ainsi, disait-il, quand on apprend à connaître nos ancêtres spirituels. Leur portrait réel est très éloquent et n'a pas besoin d'être retouché. La vraie sainteté, c'est la chose la plus simple et la plus humble qui soit quand on la rencontre chez les vrais saints. L'une des premières questions qui se posent à votre esprit au sujet du Comité des Fondateurs est de connaître quelles conditions particulières il rencontre dans la propagande en faveur des pionniers de la foi chez nous. Nous étudierons ensemble les grandes lignes du mandement épiscopal, dont l'exécution fut confiée en 1942 au Comité des Fondateurs de l'Eglise du Canada, puis nous verrons comment le Comité concilie la lettre et l'esprit de ce mandement, vingt-deux ans après son émission, dans le contexte actuel où nous vivons.

La lettre pastorale collective de NN.SS. les archevêques et évêques de la province de Québec, datée du 25 juillet 1942, prescrit une croisade de prières en faveur de la béatification et de la canonisation des Fondateurs de l'Eglise du Canada. En voici quelques extraits significatifs : « Jamais peut-être, dans l'histoire du monde, ne vit-on tant d'âmes saintes réunies autour du berceau d'un peuple. Il semble que Dieu ait voulu faire en un demi-siècle au Canada ce qu'il n'a fait ailleurs qu'en de longues périodes d'histoire...» Et nos pasteurs ajoutent dans leur lettre : " mais, depuis des années, on attend des miracles qui soient le témoignage divin nécessaire pour bien asseoir le verdict de l'Eglise. Et ces miracles Dieu les accorde quand Il Lui plaît à la prière fervente des fidèles... Pour sanctionner de son autorité souveraine l'exaltation des saints fondateurs de notre pays, il semble que le Dieu de toute 
sainteté attende la prière unanime de tout notre peuple, de toute la nation canadienne. $D$

Le mandement précise quelques paragraphes plus loin : * Nous avons besoin de leur gloire, car il n'est pas sans profit pour nous de redire au monde, et de considérer nous-mêmes de quelle noblesse chrétienne nous sommes. Nous avons besoin qu'ils nous soient proposés en modèles. Leur vie d'abnégation nous enseignera que la vraie grandeur d'un peuple, ce sont ses valeurs spirituelles; elle nous redira que le seul bur digne d'une vie chréiienne esi de faire frucifírer les irésurs de vie spirituelle que Dieu offre aux âmes... »

Ces textes expriment clairement le désir et le souci pastoral de l'épiscopat. Plus loin dans leur mandement, NN.SS. les évêques jettent les bases du Comité des Fondateurs : "Afin donc,..., d'assurer le succès de cette croisade nationale de prières, il nous a paru bon de constituer un Conseil ou Comité général de propagande, qui ait la haute direction de la campagne. Ce Comité sera présidé par le prélat que nous aurons désigné comme le représentant attitré de l'Episcopat; il comptera un certain nombre de conseillers, dont l'un agira comme secrétaire. Sous l'autorité de ce même Comité, un bureau central de propagande, organe exécutif du Comité, assurera la mise à exécution de ses directions et mots d'ordre. Centre de publicité, il organisera la diffusion de la dévotion à nos saints fondateurs par la prédication et par tous les moyens de propagande : journaux, revues, tracts, brochures. radio, cinéma et le reste. Centre de documentation, il servira à alimenter et la prédication et la propagande, grâce aux plans de sermon ou d'étude et aux autres instruments de travail qu'il fournira. Centre de compilation enfin, il tiendra registre des faveurs attribuées à l'intervention de nos saints fondateurs et, dans les limites de la prudence et de la discipline canonique, d'accord avec les Vice-Postulations, il pourra les publier à bon escient." Tel est l'essentiel du texte de la lettre pastorale suivi des instructions complémentaires du mandement.

Le Comité exaltera nos pères dans la foi qui vécurent à l'époque de nos origines et qui sont admirés par toute la nation canadiennefrançaise. L'intérêt que suscitera leur cause de postulation dépassera donc les cadres d'un diocèse ou d'une collectivité religieuse. Parmi les saints personnages du début de la Nouvelle-France, le choix de NN.SS. les évêques s'arrêta sur $\mathbf{M}^{\mathrm{gr}}$ de Laval, Marie de l'Incarnation, Catherine de Saint-Augustin et Marguerite Bourgeoys. Leur cause était déjà rendue devant les tribunaux romains, lorsqu'on confia aux soins du Comité des Fondateurs de l'Eglise du Canada, les causes de Jeanne Mance et de Marguerite d'Youville.

Le mandement stipule aussi que le secrétariat, organe exécutif du Comité superviseur, est confié aux Pères Jésuites, qui ont participé aux premiers progrès de l'Eglise canadienne et qui réunissent les qualités et les moyens pour réussir la campagne de prière. 
Les Vice-Postulations de chacune des causes conservent leur liberté d'action dans leur sphère respective, mais elles offriront leur part de collaboration aux suggestions et aux initiatives du Comité.

Toutes les paroisses et toutes les institutions organiseront des journées propres à rappeler le souvenir de nos Pionniers de la foi, par des prédications appropriées et par des cérémonies capables de raviver la piété des fidèles à leur endroit.

Les écoles, les collèges et autres maisons d'éducation devraient dispenser un enseignement exact et bien vivant sur nos ancêtres spirituels.

Nosseigneurs les évêques recommandent aussi que la croisade de prières envers nos Fondateurs s'accompagne d'une offensive de pénitence, par la pratique du jeûne et de l'aumône.

Voilà quelles étaient les principales suggestions précises et concrètes que recommandait la lettre pastorale de l'épiscopat. Six ans avant l'émission du mandement que nous venons d'analyser, soit en mai 1936, Son Eminence, le regretté cardinal Villeneuve, préconisait déjà la glorification des grands serviteurs de Dieu en notre pays. Il adressait alors la parole aux membres de notre Société réunis en congrès à Québec. A cette occasion, son Eminence se penchait sur le problème des jeunes, sur leurs aspirations. Il disait alors ces paroles, expression d'une vision profonde: «Une jeunesse se lève qui veut faire grand, selon la poussée que lui imprimeront ses maîtres et les exemples qu'on lui montrera. La conscience publique s'éveille aussi. En face des problèmes vitaux qui se posent pour la régénération ou la mort de notre société, une effervescence s'agite, qui peut inquiéter, mais qui peut aussi être le signe d'une force et d'une transformation heureuse, selon le ferment qu'on y jettera en plus grande abondance. L'élite morale et intellectuelle du pays, pour tenir, a besoin de considérer des âmes magnanimes et d'apprendre comment on agit aux grandes heures de l'humanité. C'est le cas de le rappeler avec Bossuet : « Quand l'histoire serait inutile " aux hommes, il faudrait la faire lire aux princes. Par les princes, entendons aujourd'hui tous ceux qui ont une part, si minime soit-elle, à l'administration, au gouvernement de l'Eglise et de l'Etat. Ils apprendront qu'on n'aboutit à de grandes œuvres qu'avec de hautes idées et de sublimes vertus. L'histoire de nos saints élargira l'horizon qu'il faut regarder même quand on a fait l'obscur geste quotidien. A une heure où, pour sauver l'ordre social et nos pays, il ne faut rien moins que des saints, ce sont nos saints qui nous apprendront à le devenir. "

Nous sommes à ce tournant de notre histoire où nous nous acheminerons vers la décadence ou bien vers le réalisme soulevé par l'idéal. Ça bouge au pays du Québec, nous le savons tous. Notre nation traverse une phase de transformations profondes.

Quelle place alors pourraient bien occuper les Fondateurs de l'Eglise du Canada dans nos préoccupations présentes, nationales et chrétiennes? 
Dans le grand public, le travail de publicité du Comité en faveur de nos Pionniers de la foi rencontre une certaine résistance. A notre époque de vitesse vertigineuse, où toute l'attention se concentre sur l'instant présent, où l'immédiat triomphe, sans égard pour les leçons du passé, seules les figures des grands personnages émergent de l'oubli. Il nous faut donc prouver à nos contemporains que nos ancêtres spirituels sont au nombre des gloires religieuses et nationales qui illuminent encore l'existence de notre peuple. Nous comparons alors nos héros de la sainteté à des astres lointains dont nous recevons encore la lumière. Nous démontrons qu'ils éclairent notre époque, tels des rayons de Dieu, par leurs exemples et par leurs œuvres. Les Pionniers de notre foi, dont nous désirons tous la canonisation, sont vraiment des héros de la sainteté capables de nous remuer, de nous décider à reproduire dans nos vies les qualités et les vertus qui brillent dans leurs âmes.

Le présent concile ${ }^{1}$, avec l'aide du Saint-Esprit, étudie les moyens de réaliser les améliorations nécessaires au progrès de la foi et de la sainteté dans les âmes. Nos ancêtres spirituels ont vécu ces deux mystères de grâce et peuvent sûrement nous aider à mieux accueillir la foi, à bien progresser dans la voie de la sainteté.

Le travail du Comité des Fondateurs ne peut rien ajouter au bonheur céleste des "saints" de chez nous; personne, parmi nous, ne peut rien faire pour eux à l'heure actuelle. Mais eux, par contre, peuvent faire beaucoup pour chacun de nous, dans notre acheminement vers le ciel, par leur exemple et par leur puissance d'intercession. Bénéficiaires de leurs dons, nous n'avons pas de meilleurs guides ni de meilleurs protecteurs pour plaider auprès de Dieu nos intérêts, à nous Canadiens, auxquels ils sont unis par tous les travaux de leur existence. Voilà pourquoi la glorification de nos ancêtres spirituels demeure toujours d'actualité.

Cependant, certaines fausses conceptions de la sainteté trouvent asile dans trop d'esprits. Elles paralysent la dévotion populaire que le Canada devrait avoir pour ses Pionniers de la foi. Plusieurs pensent que leur propre vie ne correspond pas beaucoup à celle de nos Fondateurs. C'est souvent parce qu'ils imaginent la sainteté comme quelque chose de merveilleux, d'irréel, comme une fièvre bienfaisante qui se développe chez certains personnages privilégiés et leur fait accomplir de grandes choses. Ils attendent que cette contagion bénie les atteigne, sans rien faire pour que la vertu progresse dans leur âme. D'autres considèrent la sainteté comme une échéance qui les surprendra bien en temps et lieu, probablement quand ils seront vieux. D'autres, enfin, s'en méfient plus que d'une tentation.

En face de ces fausses conceptions, notre propagande ne doit rien brusquer parce qu'elle touche l'âme humaine et que les vertus sont comme des fleurs : inutile de tirer sur les tiges, elles ne poussent pas plus vite. Un bon jardinier compte sur la Providence et avec le temps.

1 Vatican II. 
Il sème, il arrose ses plantes, et ne craint pas de tuer les insectes et d'arracher les mauvaises herbes. Nous agissons de même pour que nos ancêtres spirituels soient mieux connus et aimés. Nous alimentons les organes d'information en textes documentaires et même spirituels afin de rejoindre toute notre population.

Nous devons répéter souvent que la sainteté se trouve en pleine vie humaine, dans la fidélité constante au devoir quotidien. C'est se méprendre étrangement, disons-nous, que de la croire réservée à une classe particulière de surhommes ou de cadavres ambulants. La sainteté n'est pas une auréole pour un être qui n'a plus rien d'humain. Elle est accessible à toutes les âmes qui ont l'énergie de vivre intensément leur christianisme. Nous présentons les grandes figures de l'épopée mystique de nos origines comme des humains vivant dans leur temps et dans leur milieu. Nous analysons à la lumière de l'histoire certains traits de leur personnalité, certaines circonstances de leur vie. C'est ainsi que nos contemporains réaliseront que nos Fondateurs ont résolu les problèmes de tous les jours, ce qui ne les a pas empêchés de mettre leur vie et leurs mœurs en accord avec les valeurs spirituelles. Vus de cette façon, nos Pionniers de la foi deviennent sympathiques aux âmes qui cherchent la lumière et l'amour. En les connaissant mieux, les gens les aiment plus et les vénèrent tout naturellement. Voilà le contexte dans lequel nous travaillons et pourquoi le Comité des Fondateurs adapte son œuvre à la mentalité de notre milieu et de notre époque. Comment s'y prend-il ? D'abord, il procède de façon discrète et stimulante à la fois; puisqu'il s'agit de rappeler les mérites des grands serviteurs de Dieu. Mais il doit aussi s'adapter à la nature humaine et atteindre le public suivant le processus de la mémoire : impression, répétition, analogie.

Pour être efficace dans le public, toute propagande doit se soucier de créer l'impression. Elle ne doit pas craindre ensuite de se répéter, même si quelques-uns y voient une insistance irritante. De plus, elle doit trouver analogies, comparaisons et oppositions, pour rendre son message plus vivant. Ce sont là, je pense, des principes susceptibles de faire mieux connaître, vénérer et prier les Pionniers de la foi qui vécurent il y a trois siècles sur notre sol canadien.

Quels moyens emploie le Comité des Fondateurs pour atteindre ce but? Comme chacun le sait, le meilleur outil social des hommes pour communiquer entre eux demeure la parole, ou bien l'écriture qui transpose la parole de façon durable. Notre Comité fait donc connaître nos ancêtres spirituels par des conférences et des causeries. Nous nous préoccupons aussi de l'audience que nous offrent les publications et les ondes de la radio et de la télévision.

En ce qui regarde l'écrit, le Comité des Fondateurs, les centres de postulation et les sanctuaires de Bonsecours et de Varennes, qui rappelent le souvenir de nos deux bienheureuses, distribuent continuellement dans le clergé, les institutions et le grand public, les imprimés 
utiles à l'accroissement de la dévotion à nos vénérés Fondateurs. Lors de la journée de l'Eglise canadienne dans les écoles, le Comité distribue plusieurs centaines de mille feuillets, images, affiches, dessins à colorier, exercices scolaires. La génération montante fait ainsi plus ample connaissance avec ses ancêtres spirituels et les jeunes rappellent la mémoire de nos Fondateurs à leurs parents, en revenant de l'école. Les adultes peuvent se procurer des livres, des brochures, des photos et des cartes postales, des images, feuillets de prières et fascicules.

Les organes d'information, les ondes do la radio et de la télécisisioñ nous accordent une bonne collaboration, si l'on tient compte du fait que nous ne pouvons pas toujours composer un communiqué différent, adapté à chacun d'entre eux.

Pour résumer, disons que les organes d'information tiennent le public en haleine sur le sujet de nos ancêtres spirituels. Ils fourniront encore plus d'éléments pour les mieux connaître si la ferveur populaire se développe à l'endroit de nos devanciers dans la foi, et si nous les alimentons mieux de faits intéressants qui soulignent la présence de nos Fondateurs dans nos vies.

Le Comité compte dix-sept membres. Son Excellence $\mathbf{M}^{\text {gr }}$ Percival Caza est intimement lié au Comité en sa qualité de membre de l'assemblée épiscopale spécialement chargé des affaires des Fondateurs. Notre historien national, M. le chanoine Lionel Groulx, président, soutient l'élan de tous ceux qui se dévouent à la glorification des Pionniers de la foi chez nous. Les communautés religieuses d'hommes, qui ont diffusé le message évangélique aux premiers Canadiens et aux Indiens du $\mathrm{XVII}^{\mathrm{e}}$ siècle, sont représentées au sein du Comité. Le secrétariat est confié aux soins des Pères Jésuites tel que le recommande la lettre pastorale. Le père Emile Gervais est le secrétaire méritant du Comité depuis vingt-deux ans. Le père Gérard Tremblay, directeur national de l'Apostolat de la prière, en est le trésorier actuel. Les six centres de postulation conservent leur autonomie en ce qui regarde leur cause respective. Ils ont leur représentant au sein du Comité qui sert à ces centres d'agent de liaison, de conseiller et de supporteur financier. Chaque archidiocèse délègue aussi son représentant.

L'exaltation des Pionniers de la foi chez nous est un objectif qui ne manque pas de grandeur. Il demande beaucoup de dévouement de la part des responsables de sa réalisation. Ceux-ci travaillent avec ferveur à l'avancement des causes de postulation. Ils ont même le mérite de puiser dans leurs loisirs les heures qu'ils consacrent à la glorification des premiers grands serviteurs de Dieu au Canada.

Les religieuses des centres de postulation et le personnel laïc du secrétariat sont aussi très attachés à la mémoire de nos Fondateurs. Ils forment une équipe de propagandistes qui besognent à faire mieux connaître les figures attachantes de notre épopée mystique. Devant les figures attachantes de nos Fondateurs, notre âme prend ses ailes, nos facultés se déploient et jettent la semence. Cependant, distingués audi- 
teurs, que votre prudence d'historien se rassure; nous vérifions aux sources avant de nous lancer.

En ce qui me concerne, mon rôle de publiciste consiste à faire connaître nos pionniers bien plus qu'à vouloir impressionner la galerie par toutes sortes de leçons particulièrement édifiantes que je pourrais imaginer de leur vie. Nos ancêtres spirituels offrent une réalité constante, une trilogie commune dans chacune de leur vie; la prière, l'exemple et l'action. Ce sont encore de nos jours les moyens les plus certains de pratiquer la charité dans la vérité, et de correspondre à la grâce divine qui habite dans les âmes qui savent l'accueillir.

En priant, en donnant l'exemple et en agissant comme nos ancêtres spirituels, l'apôtre moderne rayonnera vraiment dans son milieu. Il se branchera sur Dieu par la prière et la grâce animera son exemple et fécondera son action. Il saura que lui-même a besoin de Dieu beaucoup plus que Notre-Seigneur a besoin de lui. Comme nos Pionniers, il travaillera pour faciliter l'avènement du Royaume en sachant bien que le sommet du christianisme n'est pas à venir, mais en comptant sur le Christ et sa grâce. Voilà le grand enseignement que je vois dans la vie de nos ancêtres spirituels, et ma tâche consiste à le répéter à mes compatriotes. Je dois le faire en me plaçant dans un triangle et regarder nos ancêtres spirituels sous trois angles : d'abord il me faut analyser les faits et gestes qui prouvent leur vertu; puis voir les vérités éternelles qu'il illustrent par leur vie; enfin trouver comment nos propres vies peuvent profiter de leurs exemples. Pratiquer cette vision triangulaire n'est pas un travail de publicité facile, mais il est très enrichissant.

Je termine maintenant cet entretien. J'espère que ma lanterne de propagandiste ne vous semble pas jeter une trop faible lueur. De toute façon, je me recommande à votre indulgence. Permettez-moi de vous dire que je suis heureux d'être au service de personnages glorieux et puissants tels que nos Fondateurs de l'Eglise du Canada. En comprenant mieux l'exemple de leur vie, j'espère progresser en science et en vertu. Comme vous tous, je souhaite bien rejoindre, dans un avenir pas trop prochain, ces glorieux personnages qui partagent maintenant ma vie entre le passé, le présent et le futur.

De ces trois temps, pour le moment c'est le présent qui importe et ma causerie deviendra bien vite chose du passé. Tout de même, j'ose espérer qu'elle vous éclairera quelque peu, lorsque notre rencontre aura pris fin.

\author{
Jacques SÉGuin \\ Publiciste au Comité des Fondateurs \\ de l'Eglise du Canada \\ Montréal, P.Q.
}

